\title{
Research on the controller of ship dynamic positioning system
}

\author{
Xiaocheng Liu \\ Key Laboratory of System Control and Information Processing \\ Shanghai Jiao Tong University \\ Shanghai, China \\ liuxc@sjtu.edu.cn
}

\author{
Weidong Zhang \\ Key Laboratory of System Control and Information Processing \\ Shanghai Jiao Tong University \\ Shanghai, China \\ wdzhang@sjtu.edu.cn
}

\begin{abstract}
This paper introduces the concept and system components of ship dynamic positioning system. Establishes the kinematic model of ship and the environmental interference model. Designs a practical nonlinear discrete controller according to the special characters of ship propulsion system. Finally researches the influence of different control parameters on the result, and universal guidance on choosing proper control parameters according to different needs will be given.
\end{abstract}

Keywords-dynamic positioning ;kinematic model; nonlinear control

\section{INTRODUCTION}

With the development of the marine, new types of hightech ship, such as the pipe laying ship, salvage ship, rescue ship plays important roles in marine engineering area. These new ships all equipped with dynamic positioning system.

Traditional anchoring system works by setting a number of anchors around the desired position to keep the ship fixed. With the increase of water depth, chain length and strength must also be increased, making the system heavier and more costly and whole system effectiveness drops[1].

Dynamic positioning system (DPS) can solve this problem. The advantage of DPS is that the cost will not increase with the increase of the water depth, and it doesn't need anchoring process, the system is also easy to operate. [2].

The wind, wave, and current will interference the vessel, making the control complicated and difficult. Even more special, the ship's propulsion system reacts slowly and the acceleration and velocity must not exceed the limit, also the working state shouldn't be changed too frequently.

The key content of this paper is a new designed practical controller and the research about how different control parameters affect the output. Simulations would be conducted. And according to different needs, guidance on choosing proper control parameters would be given.

\section{RESEARCH STATUS OF DYNAMIC POSITIONING SYSTEM}

\section{A. Introduction to dynamic positioning system}

DPS is basically a closed loop position control system. DPS makes a ship keep its desired position and heading or move along a desired trajectory using its own propulsion system.
The system uses various sensors to measure the ship's states andenvironmental disturbances, calculates control value and actuators generate required thrust to keep the vessel in the desired position. Figure 1 shows the basic structure of a ship equipped with DPS.

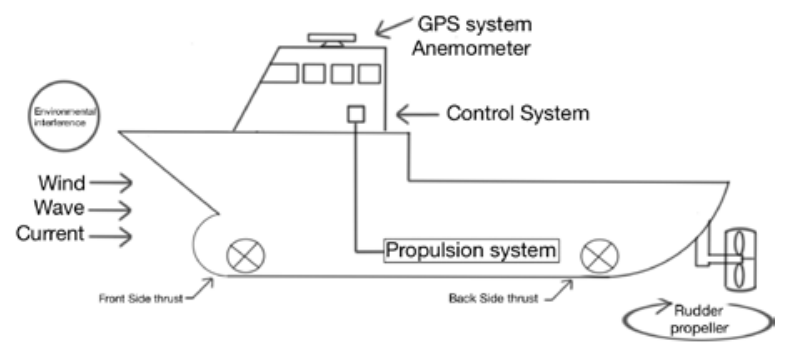

Figure 1, Schematic diagram of a ship with DPS

B. System constitution of dynamic positioning system Functionally, the DPS is divided into three subsystems:

1.) Observation system, which is responsible for measuring position, heading and other disturbs, providing information to the control system.

2.) Control system, which comprehensive the information, calculate the control values, and output to the implementation system.

3.) Implementation system, which produces thrust to keep the ship in position. It produces thrust through side thruster or rudder propeller. Response time, energy consummation and propulsion efficiency of the thruster are important parameters of the whole system.

As shown in Figure 2, a DPS is build by the following components.

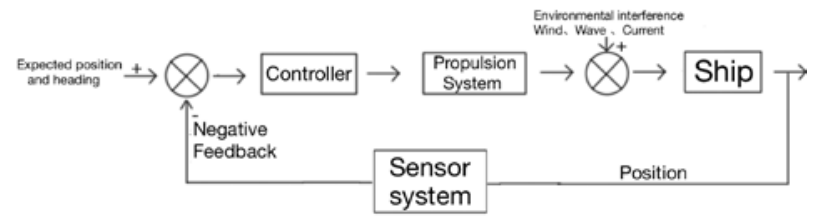

Figure 2, schematic diagram of a DP control system

\section{MATHEMATICAL MODELING}

In this chapter, we discuss about the kinematic model of the ship and the environmental interference model. 


\section{A. The kinematic model of the ship}

The ship can be considered as a rigid body, and all physical movement of the ship can be decomposed into six degrees of freedom. But practical DP systems consider only three degrees of them: surge, sway and yaw.

In order to fully describe the motion of the ship, two coordinates are established (Figure 3). They are the earth coordinate $X_{E}-o-Y_{E}$ and the local coordinate on ship $X-o-Y$. The origin point of the local coordinate coincides with the barycenter of the ship.

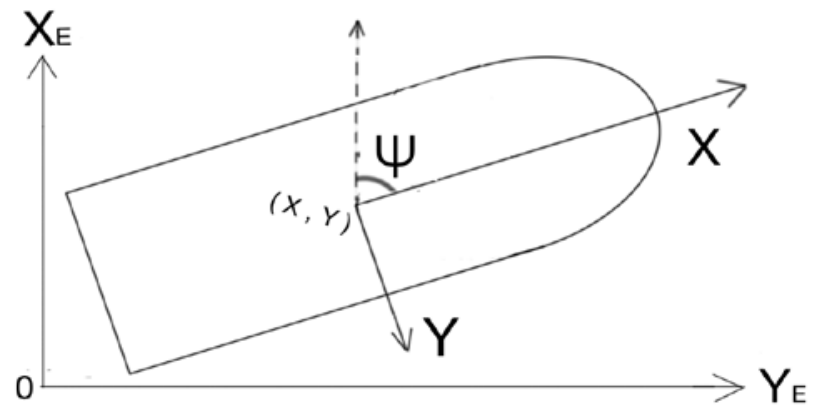

Figure 3, earth coordinate and local coordinate on ship

As shown in Figure three, the position and angle vector in the earth coordinates is $\eta=\left[\begin{array}{lll}x & y & \psi\end{array}\right]^{T}$, and the velocity vector in local ship coordinate is $v_{s}=$ $\left[\begin{array}{lll}u & v & r\end{array}\right]^{T}$.Through coordinate transformation, the following relationship is obtained [3]:

$$
\dot{\eta}=\left[\begin{array}{ccc}
\cos \psi & -\sin \psi & 0 \\
\sin \psi & \cos \psi & 0 \\
0 & 0 & 1
\end{array}\right] \cdot v_{s}(1)
$$

The kinematic model of the ship can be divided into two parts, the low frequency model and the high frequency model.

1.) Low frequency motion model:

According to [4], the low frequency motion model of the ship is as follows:

$$
M \cdot v_{\text {low }}+D\left(v_{\text {low }}-v_{\text {current }}\right)=\tau+\xi
$$

$\mathrm{M}$ is the inertia matrix of the ship, it is a positive definite matrix, $M=M^{T}>0$. Mass of the ship is $\mathrm{m}$, moment of inertia is $I_{z}$. The additional water mass caused by movement in direction Surge, sway, yaw are $X_{\dot{u}}, Y_{\dot{v}}, N_{\dot{r}}$. And the additional water mass introduction by the coupling of sway and yaw is $Y_{\dot{r}}$. D is a linear hydrodynamic coefficient matrix, and it also satisfies the positive definite requirements. In the simulation choose $\mathrm{m}=150 \mathrm{~kg}$.

$$
M=\left[\begin{array}{ccc}
m-X_{\dot{u}} & 0 & 0 \\
0 & m-Y_{\dot{v}} & m x_{G}-Y_{\dot{r}} \\
0 & m x_{G}-Y_{\dot{r}} & I_{z}-N_{\dot{r}}
\end{array}\right], D=\left[\begin{array}{ccc}
-X_{u} & 0 & 0 \\
0 & -Y_{v} & -Y_{r} \\
0 & -N_{v} & -N_{r}
\end{array}\right] \text { (3) }
$$

$v_{\text {low }}=\left[\begin{array}{lll}u_{\text {low }} & v_{\text {low }} & r_{\text {low }}\end{array}\right]^{T}$ is low frequency velocity vector. $v_{\text {current }}=\left[\begin{array}{lll}u_{\text {current }} & v_{\text {current }} & r_{\text {current }}\end{array}\right]^{T}$ is flow velocity vector. $\tau$ is the control force and torque vector, $\xi=\left[\begin{array}{lll}\xi_{u} & \xi_{v} & \xi_{r}\end{array}\right]^{T}$ is deviation, which contains the other disturbances, $\xi$ can be considered constant within a certain period of time.
Because of the coordinate transformation nonlinearityis introduced. According to the small angle theory, when the angular difference $\Delta \psi=\psi-\psi_{e}$ is small enough, the nonlinear model can be approximated with a linear model [4]:

$$
\begin{aligned}
& \dot{x}_{l}=A_{l} x_{l}+B_{l} \tau+D \xi_{l} \\
& y_{l}=C_{l} x_{l}+\gamma
\end{aligned}
$$

The state vector $x_{l}=\left[\begin{array}{lllllll}x & y & \psi & u & v & r\end{array}\right]^{T}$ contains speed and location information, $\tau$ is the control force and control torque vector, $\xi_{l}$ is the 3-D disturbance force vector, namely wind, wave and current and other disturbance. $\gamma$ is sensor measurement noise vector, which can be considered as Gauss white noise. The matrixes are defined as follows:

$$
\begin{gathered}
A_{l}=\left[\begin{array}{cc}
0_{3 \times 3} & I_{3 \times 3} \\
0_{3 \times 3} & -M^{-1} D
\end{array}\right], \quad B_{l}=\left[\begin{array}{c}
0_{3 \times 3} \\
M^{-1}
\end{array}\right] \\
D=\left[\begin{array}{l}
0_{3 \times 3} \\
M^{-1}
\end{array}\right], \quad C_{l}=\left[\begin{array}{ll}
I_{3 \times 3} & 0_{3 \times 3}
\end{array}\right]
\end{gathered}
$$

2.) High frequency motion model:

The ship's high frequency motion in the three freedom degrees can be considered having no coupling with each other. They can be seen as second-order oscillators [5].

$$
h(s)=\frac{K_{w} \cdot s}{s^{2}+2 \cdot \xi \omega_{0} \cdot s+\omega_{0}^{2}}
$$

$K_{w}$ is a parameter related to the sea, $\xi$ is damping coefficient. It is less than one. $\omega_{0}$ is the main frequency in P-M wave spectrum, related to the significant wave height. Rewriteit as matrix:

$\dot{x}_{H}=\left[\begin{array}{cccccc}0 & 1 & 0 & 0 & 0 & 0 \\ -2 \xi \omega_{0} & -\omega_{0}^{2} & 0 & 0 & 0 & 0 \\ 0 & 0 & 0 & 1 & 0 & 0 \\ 0 & 0 & -2 \xi \omega_{0} & -\omega_{0}^{2} & 0 & 0 \\ 0 & 0 & 0 & 0 & 0 & 1 \\ 0 & 0 & 0 & 0 & -2 \xi \omega_{0} & -\omega_{0}^{2}\end{array}\right] \cdot x_{H}+\left[\begin{array}{c}0 \\ k_{1} \\ 0 \\ k_{2} \\ 0 \\ k_{3}\end{array}\right] \cdot \omega_{H}$ $y_{H}=\left[\begin{array}{llllll}0 & 1 & 0 & 0 & 0 & 0 \\ 0 & 0 & 0 & 1 & 0 & 0 \\ 0 & 0 & 0 & 0 & 0 & 1\end{array}\right] \cdot x_{H}(10)$

Vector $x_{H}=\left[\begin{array}{llllll}\xi_{x} & x_{H} & \xi_{y} & y_{H} & \xi_{\psi} & \psi_{H}\end{array}\right]^{T}$ contains position and velocity state in three freedom degrees, $\omega_{H}$ is Gaussian white noise with a mean value of zero, vector $y_{H}=\left[\begin{array}{lll}x_{H} & y_{H} & \psi_{H}\end{array}\right]^{T}$ represents high frequent longitudinal swing,transverse swing and yaw angle.

$B$. The environmental interference model.

The effect of interference force from win, wave and current, can be estimated using the following model [6].

\section{1.) Wind Model}

Wind affects the ship mainly from above the waterline. Suppose Air density is $\rho_{\text {air }}$, ship forward direction projection area is $A_{x}$, lateral projection area is $A_{y}$, ship length is $\mathrm{L}$, wind speed $U_{\text {wind }}$, longitudinal wind coefficient is $C_{u x}$, transversewind coefficient is $C_{u y}$, wind torque coefficient is $C_{u m}$, the following three formula are get [7]. 


$$
\begin{gathered}
X_{\text {wind }}=\frac{1}{2} \cdot \rho_{\text {air }} \cdot A_{x} \cdot U_{\text {wind }}^{2} \cdot C_{u x} \\
Y_{\text {wind }}=\frac{1}{2} \cdot \rho_{\text {air }} \cdot A_{y} \cdot U_{\text {wind }}^{2} \cdot C_{u y} \\
M_{\text {wind }}=\frac{1}{2} \cdot L \cdot \rho_{\text {air }} \cdot A_{y} \cdot U_{\text {wind }}^{2} \cdot C_{u m}
\end{gathered}
$$

2.) Wave force model

Wave force can cause two major kinds of motion:

a.)High frequency motion caused by first order wave force. The high frequency variation caused by the first order wave force cause no drift and needs to be filtered out.

b.)Low frequency motion caused by the second-order wave force.It changes states of the ship. Suppose $C_{w x}, C_{w y}, C_{w m}$ are wave drift force coefficients, $\xi$ is effective wave height, $\theta$ is relative flow angle, ship length is $\mathrm{L}[6]$.

$$
\begin{aligned}
X_{\text {wave }} & =\frac{1}{2} \cdot \rho_{\text {water }} \cdot C_{w x} \cdot L \cdot \xi^{2} \cdot \cos \theta \\
Y_{\text {wave }} & =\frac{1}{2} \cdot \rho_{\text {water }} \cdot C_{w y} \cdot L \cdot \xi^{2} \cdot \sin \theta \\
M_{\text {wave }} & =\frac{1}{2} \cdot \rho_{\text {water }} \cdot C_{w m} \cdot L^{2} \cdot \xi^{2} \cdot \sin \theta
\end{aligned}
$$

3.) Current force model

Water current affects the ship mainly from below the waterline. Current force is tremendous. It is the main cause of draft and heading change of ship. The relative velocity is $V_{c}(m / s)$, vessel length is $\mathrm{L}$, draught depth is d. Water density is $\rho_{\text {water }}$, it will change with the temperature and the position. The relative flow angle is $\theta$, longitudinal and transverse flow force coefficient is $C_{c x}, C_{c y}$, current torque coefficient is $C_{c m}$ [6].

$$
\begin{aligned}
& x_{\text {current }}=\frac{1}{2} \cdot \rho_{\text {water }} \cdot C_{c x}(\theta) \cdot L \cdot d \cdot V_{c}^{2} \\
& y_{\text {current }}=\frac{1}{2} \cdot \rho_{\text {water }} \cdot C_{c y}(\theta) \cdot L \cdot d \cdot V_{c}^{2} \\
& M_{\text {current }}=\frac{1}{2} \cdot \rho_{\text {water }} \cdot C_{c m}(\theta) \cdot L^{2} \cdot d \cdot V_{c}^{2}
\end{aligned}
$$

\section{CONTROL Algorithms}

The most used DP algorithm is PID control algorithm [8].Butdue to the fact that the sensor output and controller are all discrete, the output $U(k)$ is actually so:

$$
\begin{array}{r}
U(k)=k_{p} \cdot e(k)+k_{i} \cdot \sum_{j=0}^{k} e(j)+k_{d} \\
\cdot[e(k)-e(k-1)]
\end{array}
$$

However, DPS has special characteristics, traditional PID control algorithms are not suitable. The reasons are as follows:

1. The DPS doesn't pursue extreme high accuracy, areasonable offset is tolerable.

2. The power consumption of the system is an important indicator, energy saving is an important consideration.
3. Propulsion system on ship can't adapt to high frequency changes in the operating conditions. Control frequency should be kept low.

Therefore, a new type of nonlinear controller is designed. It's named "Discrete nonlinear closed-loop servo positioning controller with dead zone and saturation”. This controller is practical. It is not only a pure mathematical model, but also can be used for real.Innovation points are as follows:

1. It introduces the dead zone and saturation range, so the physical limits of real equipment are taken into account.

2. It introduced control interval to prevent too high adjustment frequency of the equipment on board.

3. It considers the mismatch of the ship's intrinsic frequency and the sampling frequency of the sensor, so a exponential forgetting factor is introduced.

Because of the introduction of control value limitation, control interval and forgetting factor, anew problem arise: the traditional analysis method can't make a good quantitative analysis. In order to research how different control parameters affect the results, several simulations are conducted:

As shown in Figure 4, the frame of the simulation with this controller is as follows, it has three layers:

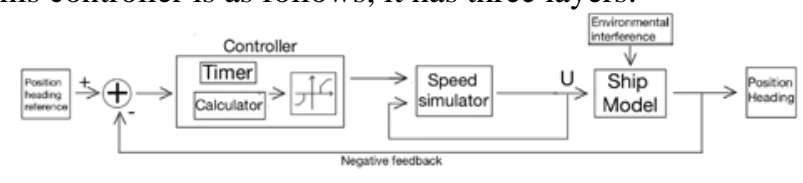

Figure 4, the simulation frame.

1. The inner layer is controller based on traditional differential PID algorithms, but many new features are introduced.

a) Due to the mismatch of ship's intrinsic frequency and the sensor sampling frequency, too long integral time could not help but have negative effect, so an exponential forgetting factor is introduced. For simulation with single step time of $1 \mathrm{~ms}, \eta$ is chosen 0.999 . Intermediate valueA(k) is as follows:

$$
\begin{gathered}
A(k)=k_{p} \cdot e(k)+k_{i} \cdot I(k)+k_{d} \cdot[e(k)-e(k-1)] \\
(12) \\
I(k)=0.999 \cdot I(k-1)+e(j), I(1)=e(1)(13)
\end{gathered}
$$

b) In order to avoid too frequent state change and too great load of the propulsion system, the dead zone and maximum acceleration limit module are introduced:

$$
\begin{aligned}
& \text { if }\left(|A(k)|<A_{\text {min }}\right) \rightarrow A_{\text {out }}(k)=0 \\
& \text { if }\left(|A(k)|>A_{\text {max }}\right) \rightarrow A_{\text {out }}(k)=A_{\text {max }} \\
& \text { if }\left(|A(k)|<-A_{\text {max }}\right) \rightarrow A_{\text {out }}(k)=-A_{\text {max }}
\end{aligned}
$$

c) The new added Timer controls the output frequency of the whole control system, preventing too frequent changes, so the actuators are effectively protected.

The addition of these control strategies makes the controller more realistic and closer to the actual situation, but also introduces nonlinear factors, which makes the analysis more complex. 
2. The intermediate layer is the velocity simulation layer, which simulates the ship's speed. It takes the hydrodynamic resistances into account, and makes proper correction, so that the simulation is closer to the real situation.

3. The outer layer is the position layer, which produces the final result of the simulation. The final results also feedback to the controller to generate control value. Random disturbances are added to this layer to make the simulation closer to the real thing.

\section{CONCLUSIONS}

This chapter simulates a process, in which the ship move from 0 meter to 5 meter and keep that position using its DP system in the longitudinal direction. The position offset and power consumption of the DP system are important indicators. The effect of different control interval and the size of the thrust dead zone are researched.

Simulation with different control interval (1 second,2 seconds and 5 seconds) and different thrust dead zone (0N,5 N and $10 \mathrm{~N}$ ) are conducted. The results are analyzed from different aspects, such as minimum energy consummation, working time as long as minimum offset. Finally a universal guidance on parameter selection is given.

A. Analyze the influence of different control intervals on the system. In order to make the difference clear, no thrust dead zone is set.

\section{1.) Control interval is 1 second, no thrust dead zone.}
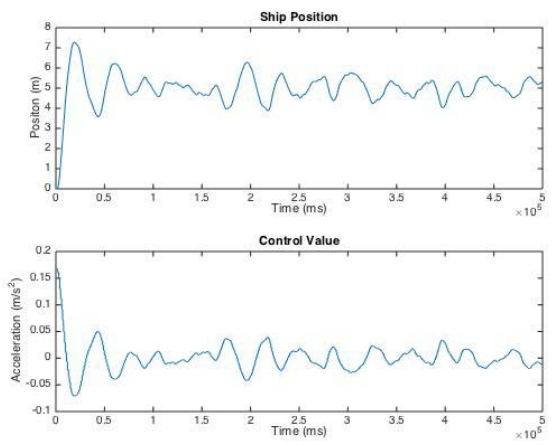

From the picture above, when control interval is 1 second, the offset of position is less than 1 meter, and the acceleration of the system is within $0.03 \mathrm{~m} / \mathrm{s}^{2}$.

2.) Control interval is 2 seconds, no thrust dead zone.
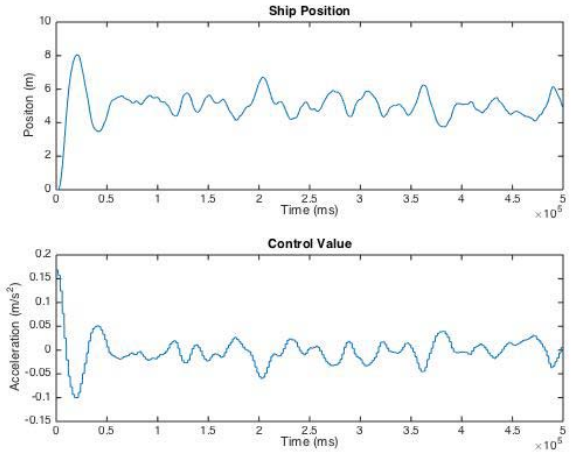

From the image above, when control interval is 2 seconds, the offset of position is less than 1.2 meter, and the acceleration of the system is within $0.05 \mathrm{~m} / \mathrm{s}^{2}$.

3.) Control interval is 5 seconds, no thrust dead zone.
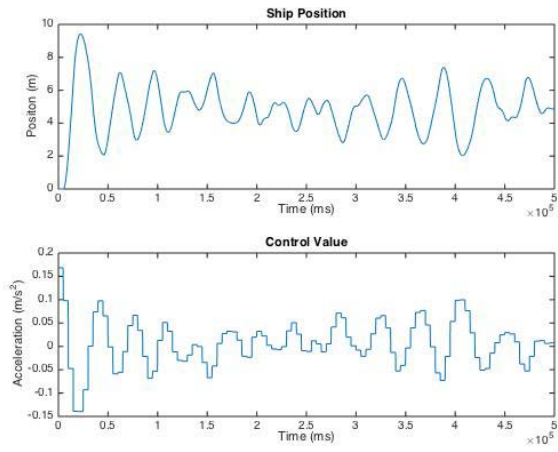

From the image above, when control interval is 5 seconds, the offset of position is less than 3 meter, and the acceleration of the system is within $0.1 \mathrm{~m} / \mathrm{s}^{2}$.

Difference of the above three cases are compared, shown in the following table:

Table 1, Result with different control interval

\begin{tabular}{|c|c|c|c|}
\hline Controlinterval & $1 \mathrm{~s}$ & $2 \mathrm{~s}$ & $5 \mathrm{~s}$ \\
\hline max. off set & $1 \mathrm{~m}$ & $1.2 \mathrm{~m}$ & $3 \mathrm{~m}$ \\
\hline max. acceleration & $0.05 \mathrm{~m} / \mathrm{s}^{2}$ & $0.05 \mathrm{~m} / \mathrm{s}^{2}$ & $0.1 \mathrm{~m} / \mathrm{s}^{2}$ \\
\hline
\end{tabular}

With the increase of the control interval, the effect of control is significantly worse. The difference between 1 second and 2 seconds is not big, but when the control interval is 5 seconds, the error of 3 meters is much larger.

The accuracy of general GPS system is about a few meters, and the accuracy of differential GPS is about 1 meter. And normal positioning accuracy requirement of real DPS is about 3 meters, so it can be considered that a control interval of 1-2 seconds is appropriate.

B. Analyze the influence of different thrust dead zone. Control interval is set to 2 seconds.

1.) Control interval is 2 seconds, no thrust dead zone. 

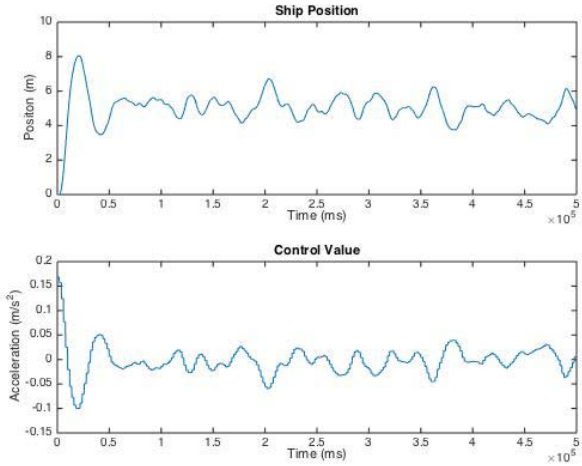

From the image above, when control interval is 2 seconds, and the thrust dead zone is 0 Newton, the offset of position is less than 1 meter, and the acceleration of the system is kept within $0.05 \mathrm{~m} / \mathrm{s}^{2}$. Control time accounts for $100 \%$ of the total time. The adjustment is quick.

2.) Control interval is 2 seconds, with a thrust dead zone of 5 Newton.

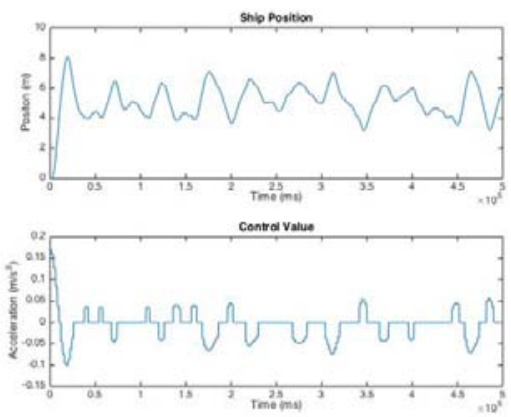

From the image above, when control interval is 2 seconds and the thrust dead zone is 5 Newton, the offset of position is less than 1.9 meter, the acceleration is kept within $0.075 \mathrm{~m} / \mathrm{s}^{2}$, control time accounts for about $40 \%$ of the total time. The adjustment is relative quick.

3.) Control interval is 2 seconds, with a thrust dead zone of 10 Newton.
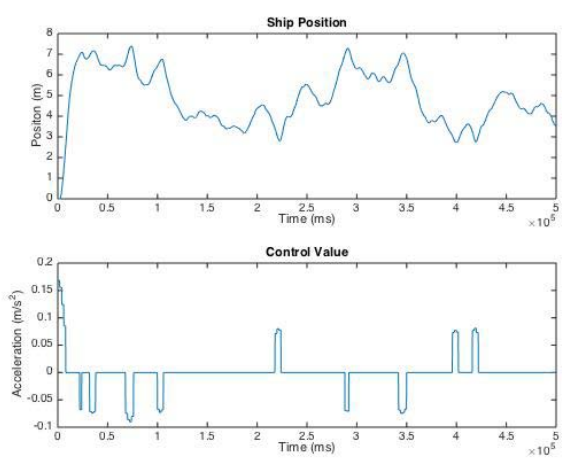

From the image above, when control interval is 2 seconds and the thrust dead zone is 10 Newton. The offset of position is less than 2.2 meter, the acceleration is kept within $0.09 \mathrm{~m} / \mathrm{s}^{2}$, control time accounts for about $10 \%$ of the total time. The adjustment is relative slow.
Difference of the above three cases are compared, shown in the following table:

Table 2,Control with different thrust dead zone

\begin{tabular}{|c|c|c|c|}
\hline thrustdeadzone & $0 \mathrm{~N}$ & $5 \mathrm{~N}$ & $10 \mathrm{~N}$ \\
\hline max.offset & $1 \mathrm{~m}$ & $1.9 \mathrm{~m}$ & $2.2 \mathrm{~m}$ \\
\hline max.acceleration & $0.05 \mathrm{~m} / \mathrm{s}^{2}$ & $0.075 \mathrm{~m} / \mathrm{s}^{2}$ & $0.09 \mathrm{~m} / \mathrm{s}^{2}$ \\
\hline ControlTime & $100 \%$ & about $40 \%$ & about $10 \%$ \\
\hline
\end{tabular}

As the table clearly shows, with the same control interval, in these cases 2 seconds, when the thrust dead zone increase from 0 Newton to 5 Newton and then to 10 Newton, the offset of position rose from within 1 meter to around 2.2 meters. Control quality is obviously decreased, and maximum acceleration also increases.

But the DPS has special requirements, it doesn't need very high accuracy, offset within requirement is enough. If the result satisfies the prerequisites, control interval and thrust dead zone can be increased, so that better energy consummation is achieved and the time of control can be significantly reduced so the mechanical wear is reduced. Since the mass of the model is set to $150 \mathrm{~kg}$, a thrust of $5 \mathrm{~N}$ corresponds to the acceleration of $0.033 \mathrm{~m} / \mathrm{s}^{2}$.

Summary all the simulation results, a general conclusion can be given:

\section{1.) About control interval}

Control interval is not the smaller the better. Too small control interval has good effect, but cause the propulsion system to wear too quickly, and too large control interval can't meet the control needs. Therefore, according to practical propulsion system selecting a suitable control interval is very important. According to the simulation can be considered that the control interval of 1-2 seconds is a suitable value for an actual ship.

\section{2.) About thrust dead zone}

No thrust dead zone means the propulsion system is in constant change, causing unnecessary energy losses and cause wear. And too large thrust dead zone cause too large offset, and makes the propulsion system works in a relatively high load situation, but the condition is stable, and fewer changes reduce the wear.Therefore, the exact thrust dead zone should be chosen based on specific circumstances.

According to the simulations, the boundary value of thruster dead zone should be chosen, so that the accelerations of the ship equal $0.03-0.06 \mathrm{~m} / \mathrm{s}^{2}$.

\section{REFERENCES}

[1] ZHAO Zhigao,YANG Jianmin,WANG Lei,et al.The development and research method of dynamic positioning system [J].The Ocean and Engineering, 2002, 20(1): 91-97 
[2] Zhou Li,Wang Lei,Chen Heng.Review on the study of dynamic positioning control system for vessels [J].Ship \& ocean Engineering,2008,37(2): 86-91

[3] Tong Jinjun,He Liming,Tian Zuohua.The mathematical model of ship with dynamic positioning system[J].Ship engineering,2002,5:27-29

[4] T. Fossen. Guidance and control of ocean vehicles. New York: Wiley, 1994.

[5] Wang Yuanhui, Shi Xiaocheng,Bian xianqian. Dynamic positioning control method of Ship based on model predictive control[J]. Ship engineering,2007,29(3),22-25
[6] Han bing, Dong Shengli. Control Algorithm Design of SRIVC2110DP Dynamic Positioning System. journalofshanghaiship and shipping research institute, 2011,34(2),113-118

[7] Wang Zhidong, $\mathrm{Xu}$ Yuru. Prediction of the motion performance of the ship under the action of wind and flow [J]. Journal of East China Ship building Industry College.1995,9 (2): 1-6

[8] Fu Mingyu, Ding Fuguang, Bian Qian. The design and research of filter for ship Dynamic Positioning System [J]. ship engineering, 1996,11(6):43-46 